\title{
Short-Term Administration of Antivascular Endothelial Growth Factor Antibody in the Late Follicular Phase Delays Follicular Development in the Rhesus Monkey*
}

\author{
RALF C. ZIMMERMANN, ENNIAN XIAO, NABIL HUSAMI, MARK V. SAUER, \\ ROGERIO LOBO, JAN KITAJEWSKI, AND MICHEL FERIN \\ Departments of Obstetrics and Gynecology and Psychiatry, and Center for Reproductive Sciences, \\ Columbia University College of Physicians and Surgeons, New York, New York 10032
}

\begin{abstract}
Indirect evidence in the nonhuman primate and human suggests that angiogenesis and regulators of angiogenesis such as vascular endothelial growth factor (VEGF) may play an active role in cyclic folliculogenesis. Indeed, the follicle selected for maturation and ovulation possesses a denser microvascular network, and VEGF messenger ribonucleic acid and its protein have been identified in granulosa cells of the developing follicle during the mid- and late follicular phases, with a more intense signal in the mature follicle. The objective of this study was to obtain direct evidence in the nonhuman primate for an active role of VEGF in follicular growth and maturation by studying the effect of VEGF-blocking antibodies in this process. After documenting two normal ovulatory cycles, female rhesus monkeys $(\mathrm{n}=7)$ received iv injections of anti-VEGF antibodies $(0.5 \mathrm{mg})$ twice on successive days in the late follicular phase. Three monkeys also received nonspecific goat IgG $(0.5 \mathrm{mg})$ twice on successive days in the late follicular phase. Daily measurements of estradiol, progesterone, $\mathrm{LH}$, and FSH were obtained during the two control cycles, the antiVEGF treatment and posttreatment cycles, and the IgG treatment cycle. Anti-VEGF antibody administration significantly lengthened
\end{abstract}

the follicular phase in six of seven monkeys to $17.8 \pm 1.7 \mathrm{vs} .10 .0 \pm$ 0.7 and $9.8 \pm 0.6$ in control cycles and $10.7 \pm 0.3$ days (mean $\pm \mathrm{SE}$ ) in IgG-treated cycles. The expected late follicular phase rise in estradiol, as documented in the control cycles (day 0,96.1 \pm 6.0 ; day 1 , $125.5 \pm 20.0$; day $2,165.5 \pm 24.9$; day $3,183.8 \pm 11.0 \mathrm{pg} / \mathrm{mL}$ ), was interrupted by anti-VEGF antibody treatment $(99.3 \pm 5.0$, day 0 , preinjection control) to $63.3 \pm 12.2$ (day 1 ), $48.5 \pm 8.7$ (day 2 ), and $57.6 \pm 9.0$ (day 3). Mean FSH levels were significantly increased by day 2 of anti-VEGF antibody treatment. After a variable delay, estradiol concentrations increased to reach a preovulatory peak in all anti-VEGF-treated animals, followed by ovulation, normal luteal function, and a normal posttreatment cycle. The data clearly demonstrate that short-term inhibition of angiogenesis with an antiVEGF-blocking antibody during the later growth phase of the dominant follicle interferes with normal follicular development. Persistence of estradiol secretion and delayed resumption of its rise also suggest recovery of the follicle. We conclude that the angiogenic regulator VEGF is a crucial component in the process of follicular growth in the primate. (J Clin Endocrinol Metab 86: 768-772, 2001)
$\mathrm{P}$ YYSIOLOGICALLY, ANGIOGENESIS in the adult female is confined to the ovary and uterus, where it is active during the menstrual cycle and pregnancy (1-3). Several potential angiogenic regulators have been identified (4). One of the most important regulator is vascular endothelial growth factor (VEGF), which has been well demonstrated to exert potent angiogenic activity, both in vitro and in vivo (5-7). Indirect evidence in the primate suggests that angiogenesis and regulators of angiogenesis may play an active role in cyclic folliculogenesis. First, angiogenesis is initiated early in the development of the follicle, and a complex vascular network is formed within the thecal cell layer during follicular growth (8). Importantly, the selected follicle has been shown to possess a denser microvascular network than that of lesser developed follicles, suggesting a more active angiogenic process in this follicle destined for ovulation (9).

Received June 9, 2000. Revision received October 4, 2000. Accepted October 13, 2000.

Address all correspondence and requests for reprints to: Dr. Michel Ferin, Department of Obstetrics and Gynecology, Columbia University College of Physicians and Surgeons, 630 West 168th Street, New York, New York 10032. E-mail: mf8@columbia.edu.

* This work was supported in part by the Center for Endometriosis Treatment and Research at Columbia University and NIH Grant DK-39144.
Second, in situ hybridization studies in the monkey have demonstrated VEGF messenger ribonucleic acid to be present in granulosa cells of the developing follicle during the mid- and late follicular phases, with a more intense hybridization signal in the mature follicle (10). In the human ovary, VEGF receptor is detected mostly in theca interna cells of antral and developing follicles (11-13). A greater intensity of VEGF staining is also seen in the more mature human follicle, whereas no activity is detected in primordial, primary follicles or atretic follicles. VEGF activity is well correlated with proliferating activity markers in the vascular endothelial cells $(4,7)$.

Although the above data suggest a putative role of VEGF in folliculogenesis, direct evidence for an active role of angiogenesis, and of VEGF in particular, in the cyclic follicular growth and maturation process remains to be obtained. The present study in the rhesus monkey was designed to provide such evidence by investigating the effects of a temporary suppression of angiogenesis during the late follicular phase using anti-VEGF antibodies.

\section{Materials and Methods}

\section{Animals}

Regularly cycling female rhesus monkeys (Macaca mulatta), weighing $4-8 \mathrm{~kg}$, were used in this study. The animals were housed in individual 
cages in temperature- and light (lights on, 0730-1930 h)-controlled rooms. They were fed Purina monkey chow (Ralston Purina Co., St. Louis MO) twice daily and fresh fruit or vegetables. Water was available at all times. Menstruation was determined by daily vaginal swabbing. Blood samples were obtained by venipuncture (a process to which the animals had been previously habituated). The experimental protocol was approved by the animal care and use committee of Columbia University and was performed in accordance with the NIH Guide for the Care and Use of Laboratory Animals.

\section{Experimental protocols}

The experiment was designed to investigate in the primate the role of angiogenesis in folliculogenesis using an anti-VEGF antibody. Two normal control ovulatory cycles were documented before initiation of the experiment. In the first experimental protocols, animals $(\mathrm{n}=7)$ received iv injections of anti-VEGF antibodies ( $0.5 \mathrm{mg}$ each day) on 2 successive days in the late follicular phase, at a time when mean estradiol $\left(\mathrm{E}_{2}\right)$ concentrations were $99.3 \pm 5.0( \pm \mathrm{SE} ;$ range, $74-112) \mathrm{pg} / \mathrm{mL}$. To monitor possible delayed effects of antibody treatment, the posttreatment cycle was also investigated. Daily blood samples were drawn throughout the entire period to monitor cyclicity in the control, treatment and posttreatment cycles and to determine the effects of anti-VEGF antibody treatment. After one normal ovulatory cycle was again documented, three of these monkeys received iv injections of nonspecific goat $\operatorname{IgG}(0.5 \mathrm{mg}$ each day) on 2 successive days in the late follicular phase (mean $\mathrm{E}_{2}, 97.3 \pm 4.1$; range, $91-117 \mathrm{pg} / \mathrm{mL}$ ). Daily blood samples were similarly obtained and analyzed.

Polyclonal antihuman VEGF antibody (R\&D Systems, Inc., Minneapolis, MN) was raised in goats immunized against recombinant human VEGF containing 165 amino acid residues $\left(\operatorname{rhVEGF}_{165}\right)$. This VEGFspecific IgG was purified by human VEGF affinity chromatography. [According to the manufacturer, this antibody shows no cross-reactivity with human platelet-derived growth factors (hPDGF-AA, hPDGF-AB, and hPDGF-BB), hVEGF B, hVEGF C, hVEGF D, human placenta growth factor, human connective tissue growth factor, and hFlt-1, and was selected for its ability to neutralize the biological activity of rhVEGF 165 and rhVEGF $_{121}$.] Purified goat IgG (Sigma, St. Louis, MO) was obtained from pooled normal goat sera. Both lyophilized preparations were reconstituted in physiological saline to final concentrations of $1 \mathrm{mg} / \mathrm{mL}$, and aliquots were stored at $-70 \mathrm{C}$ until use.

\section{Assays and statistical analysis}

Blood samples were centrifuged, and sera were kept at $-20 \mathrm{C}$ until assay. Concentrations of $\mathrm{E}_{2}$, to assess follicular maturation, and of progesterone, to evaluate luteal phase quality, were measured in daily serum samples by a chemiluminescent immunoassay using the Immulite system (Diagnostic Products, Los Angeles, CA). Interassay coefficients of variation were $11.9 \%$ and $11.1 \%$ for $E_{2}$ and progesterone, respectively. To detect potential effects of the treatment on gonadotropin secretion, LH and FSH concentrations were also measured by recombinant homologous RIA (14) using reagents provided by the National Hormone and Pituitary Program. Assay sensitivity (at 95\% binding) for $\mathrm{LH}$ and FSH was 0.01 and $0.02 \mathrm{ng} /$ tube respectively. Intra- and interassay coefficients of variation were $7.0 \%$ and $13.1 \%$, respectively, for $\mathrm{LH}$ and $5.0 \%$ and $6.1 \%$ for FSH.

Cycle parameters (length of the follicular and luteal phase), hormone concentrations, and integrated progesterone values during the luteal phase (as calculated by trapezoidal analysis of the areas under the daily progesterone curves) were compared in control, treatment, and posttreatment cycles. Comparisons between control and experimental cycles were made using multiple ANOVA, followed by the Tukey test. The level of significance was set at $P<0.05$.

\section{Results}

\section{Menstrual cycle length}

Anti-VEGF antibody administration in the late follicular phase significantly lengthened the follicular phase in six of seven monkeys, from $10.0 \pm 0.7$ and $9.8 \pm 0.7$ days (mean \pm $\mathrm{SE})$ in the two preceding control cycles to $17.8 \pm 1.7$ days in the treatment cycle. Posttreatment cycle length, however, was similar to that in the control cycles (Table 1). One monkey (not included in Table 1 or Fig. 1) did not respond to the treatment. The follicular phase in animals receiving nonspecific goat $\operatorname{IgG}(\mathrm{n}=3)$ was similar in length $(10.7 \pm 0.3$ days $)$ to that in the control cycle (Table 1). There were no differences in luteal phase length between control, treatment, and posttreatment cycles (Table 1).

\section{Hormonal response}

After anti-VEGF antibody administration, $E_{2}$ concentrations decreased from $99.3 \pm 5.0 \mathrm{pg} / \mathrm{mL}$ (preinjection control on day 0 ) to $63.3 \pm 12.2$ (day 1$), 48.5 \pm 8.7$ (day $2 ; P<0.01$ ), and $57.6 \pm 9.0$ (day $3 ; P<0.05 ;$ Fig. 1 ). These values contrast with the increasing $E_{2}$ concentrations observed in the same animals during the control cycles; starting on a day of comparable $E_{2}$ concentrations (day 0: $96.1 \pm 6.0$ ), these values increased to $125.5 \pm 20.0$ (day 1 ), $165.5 \pm 24.9$ (day 2 ), and $183.8 \pm 11.0$ (day 3 ). Figure 1 compares mean $\mathrm{E}_{2}$ concentrations in control cycles to individual $\mathrm{E}_{2}$ concentrations in six monkeys receiving anti-VEGF antibody. After a variable delay in the animals receiving anti-VEGF antibody, $\mathrm{E}_{2}$ concentrations increased to reach a preovulatory peak. Mean preovulatory $\mathrm{E}_{2}$ peaks were similar in control cycles (183.8 \pm $11.0 \mathrm{pg} / \mathrm{mL})$, anti-VEGF-treated cycles (230 \pm 29.8$)$, and nonspecific IgG-treated cycles $(187 \pm 17.8)$, respectively. All animals had a LH surge, although it was delayed in six monkeys treated with anti-VEGF antibody. Ovulation occurred in all animals, and although appearance of the luteal phase was delayed in animals receiving anti-VEGF antibody, luteal function, as reflected by integrated luteal progesterone concentrations, was comparable in the control, treatment, and posttreatment cycles (Table 1). Basal LH and FSH concentrations increased in all six animals after anti-VEGF antibody treatment compared with those in animals treated with nonspecific IgG, although the increase was statistically significant only for FSH (Table 2). No effect of nonspecific goat IgG administration on $\mathrm{E}_{2}$ or progesterone secretion was observed.

Figures 2 and 3 illustrate longitudinal hormone changes in the two successive control, anti-VEGF treatment, and posttreatment cycles and in the IgG-treated cycle in two individual monkeys. Treatment with the specific antibody, but not with nonspecific IgG, delayed the process of follicular maturation by 6 days in monkey RCJ2 and by 8 days in

TABLE 1. Effects of a 2-day administration of anti-VEGF antibody during the late follicular phase on menstrual cycle parameters

\begin{tabular}{|c|c|c|c|c|c|}
\hline & Control 1 & Control 2 & Anti-VEGF cycle & Posttreatment & IgG cycle \\
\hline Follicular length (days $\pm \mathrm{SE}$ ) & $10.0 \pm 0.7$ & $9.8 \pm 0.7$ & $17.8 \pm 1.7^{a}$ & $10.4 \pm 1.2$ & $10.7 \pm 0.3$ \\
\hline Luteal length (days $\pm \mathrm{SE})$ & $15.3 \pm 0.3$ & $15.4 \pm 0.4$ & $15.8 \pm 0.1$ & $15.6 \pm 0.1$ & $15.3 \pm 0.7$ \\
\hline Luteal progesterone (ng/mL) & $49.9 \pm 4.1$ & $45.5 \pm 3.1$ & $55.4 \pm 3.5$ & $40.2 \pm 4.0$ & $53.0 \pm 4.0$ \\
\hline
\end{tabular}

${ }^{a} P<0.05$ vs. controls, posttreatment, and IgG cycles. 

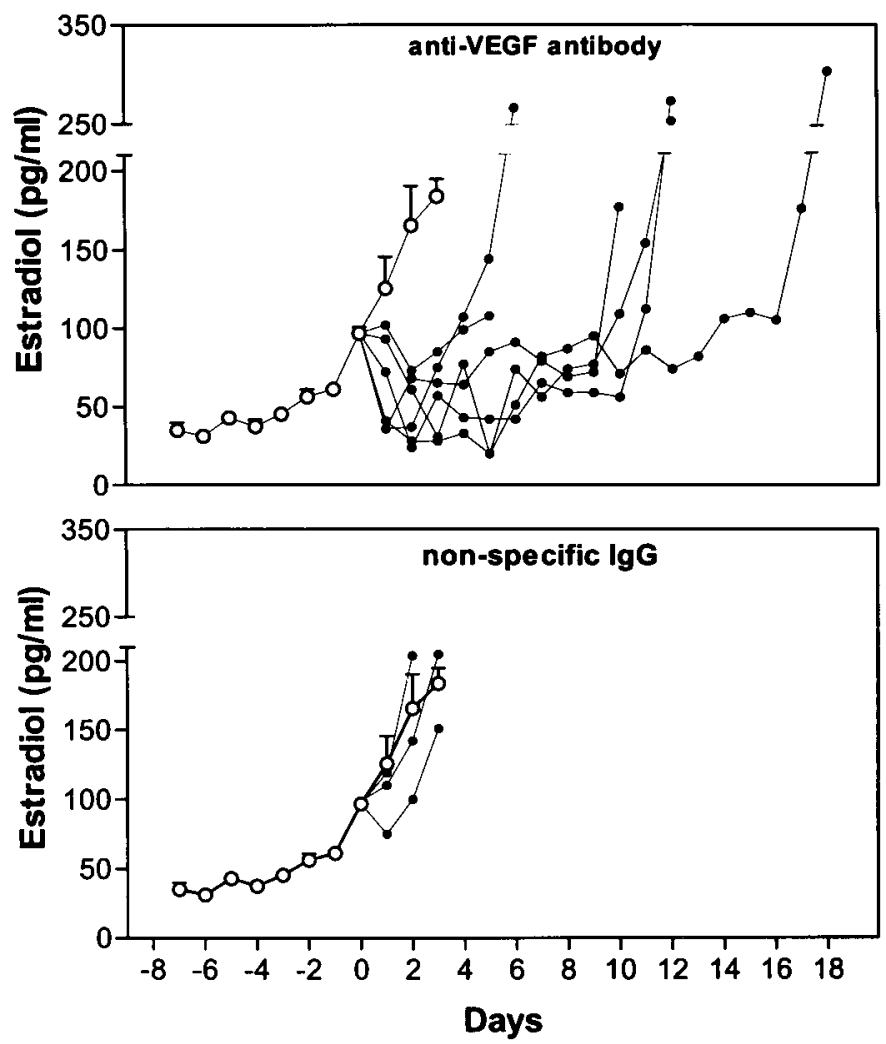

FIG. 1. Daily $\mathrm{E}_{2}$ concentrations in control cycles, anti-VEGF antibody-treated cycles (upper panel), and IgG-treated cycles (lower pan$e l$ ). Data for the control cycles are the mean $\pm \mathrm{SE}$ concentrations (open circles). Day 0 represents the first day of injection of the anti-VEGF antibody or IgG or, in the control cycles, the day of comparable $\mathrm{E}_{2}$ concentrations. $\bigcirc$, Mean of control and preinjection values. For the anti-VEGF- or IgG- treated cycles $(\bullet)$, individual $\mathrm{E}_{2}$ concentrations up to the preovulatory $\mathrm{E}_{2}$ peak are shown for six of seven monkeys responding to the anti-VEGF treatment and the three IgG-treated monkeys.

monkey RTC2 compared with the preceding control cycles in the same animals.

\section{Discussion}

Our data in the primate clearly indicate that short-term inhibition of angiogenesis after anti-VEGF antibody administration during the later growth phase of the dominant follicle interferes with its normal development. This evidence, supplemented by immunohistochemical and in situ hybridization data indicative of increasing VEGF activity (10-13) and by the manifestation of increased capillary density (9) in the maturing follicle, provides a direct demonstration of the requirement for VEGF involvement and active angiogenesis in the later stage of the normal follicular maturation process.

In this specific protocol, anti-VEGF treatment was initiated in the late follicular phase, at a time when a dominant follicle has already been selected and when peripheral $E_{2}$ secretion reflects the growth rate of this follicle (15-17). The facts that the treatment interrupted the characteristic rise in $\mathrm{E}_{2}$ secretion and resulted in a significant lengthening of the follicular phase directly demonstrate that in the absence of adequate VEGF stimulation, normal follicular maturation is interfered with. We interpret this impediment to normal follicular growth to be the result of a temporary interruption of local angiogenesis. In addition, these data may reflect a reduction in local vascular permeability, as experimental data suggest a role for VEGF in this process $(6,7)$. In either case, neutralization of VEGF activity would, among other effects, limit access to the peripheral factors needed to support follicle growth, such as, for example, the gonadotropins. It is well known that $\mathrm{LH}$ and $\mathrm{FSH}$ are required for proper $\mathrm{E}_{2}$ secretion $(15,16)$. Interestingly, data in the literature also suggest a role for the gonadotropins in VEGF production by the follicle. Studies with monkey granulosa cells demonstrate that not only are large amounts of gonadotropins representative of the midcycle ovulatory surge, but smaller amounts, more typical of tonic secretion, enhance local VEGF production (18, 19). A similar stimulation of VEGF is seen in PMS-stimulated rats after hCG administration (20). Furthermore, decreased VEGF messenger ribonucleic acid expression, albeit in the corpus luteum, is seen in the monkey after GnRH antagonist administration. Such data confirm the dependence of local ovarian VEGF activity on tonic gonadotropin secretion (10), and thus, it may not be surprising that VEGF activity increases in the maturing follicle in the late follicular phase. Overall, VEGF antibody treatment may not only impede angiogenesis directly, but also potentially decrease local VEGF production by modifying local vascular permeability and decreasing gonadotropin support of VEGF synthesis.

Similar effects on $E_{2}$ secretion and menstrual cycle length were reported in the human and the monkey after the administration of a GnRH antagonist during the follicular phase (21-23). Although this remains to be demonstrated, such an effect of GnRH antagonist treatment on the growing follicle may well partially reflect a local decrease in available VEGF. The hormonal environments after GnRH antagonist and anti-VEGF antibody treatment, however, are quite different. Gonadotropin levels are obviously suppressed after antagonist administration (22), whereas our data show a short-term increase in FSH release after anti-VEGF antibody treatment, presumably associated with a decrease in $\mathrm{E}_{2}$ levels and the parallel decline in the negative feedback activity of the steroid.

Although the normal late follicular rise in $\mathrm{E}_{2}$ is interrupted in monkeys receiving anti-VEGF antibodies, it is unlikely that the selected maturing follicle is destroyed in this process, because some degree of $E_{2}$ secretion persists. Once $E_{2}$ secretion resumes, the ovulatory LH surge follows without delay in most monkeys, as it does in the normal cycle. Thus, we speculate that anti-VEGF antibody treatment interferes temporarily with normal vascularization function in the selected maturing follicle and that this follicle can be maintained for several days in a suspended state. Data obtained after a 4-day GnRH antagonist treatment in the human also support a temporary suspension of follicle growth, as monitored by ultrasonographic measurements of follicular diameter (21, 22). Such studies remain to be performed in our experimental model.

This report on the effectiveness of an anti-VEGF antibody to interfere with normal follicular development in the rhesus parallels data in the marmoset monkey showing interference with normal luteal phase function after VEGF neutralization (24). Treatment with VEGF-blocking antibodies in the luteal 
TABLE 2. Effects of anti-VEGF or IgG treatment on gonadotropin levels

\begin{tabular}{lcccc}
\hline & Day 0 & Day 1 & Day 2 & Day 3 \\
\hline LH (ng/mL) & & & & \\
Anti-VEGF & $0.98 \pm 0.2$ & $2.3 \pm 0.5$ & $2.1 \pm 0.4$ & $1.5 \pm 0.3$ \\
IgG & $0.90 \pm 0.1$ & $0.9 \pm 0.15$ & $0.93 \pm 0.17$ & $0.93 \pm 0.1$ \\
FSH (ng/mL) & & & & \\
Anti-VEGF & $1.66 \pm 0.22$ & $1.38 \pm 0.09$ & $2.42 \pm 0.23^{a}$ & $2.72 \pm 0.29^{a}$ \\
IgG & $1.44 \pm 0.16$ & $1.10 \pm 0.04$ & $0.90 \pm 0.06$ & $0.90 \pm 0.06$ \\
\hline
\end{tabular}

The value on day 0 is the pretreatment control, after which treatment was initiated.

${ }^{a} P<0.05$ vs. IgG cycle.
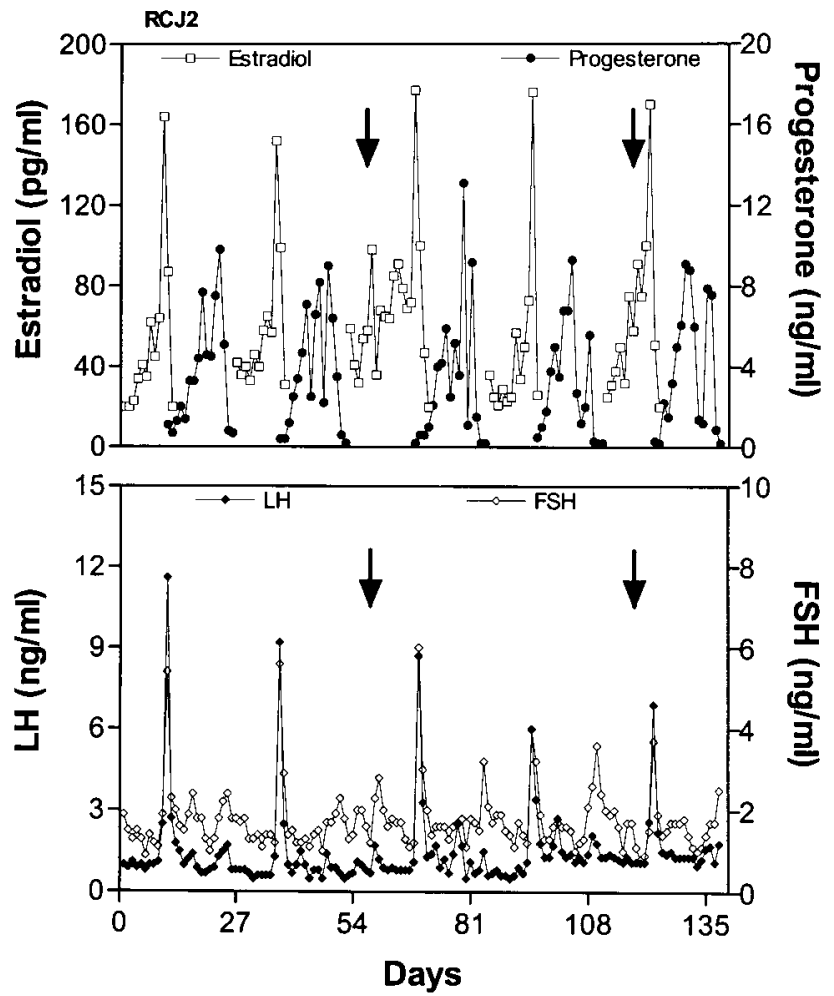

FIG. 2. Two successive control cycles followed by an anti-VEGF antibody-treated cycle, a posttreatment cycle, and an IgG-treated cycle in monkey RCJ2. Shown in the upper panel are the daily $\mathrm{E}_{2}$ and progesterone levels. The first and second arrows indicate the times of anti-VEGF antibody and IgG administration, respectively. The lower panel illustrates daily LH and FSH concentrations. Note the temporary interruption of normal $\mathrm{E}_{2}$ secretion after anti-VEGF antibody injection and the simultaneous small rise in tonic LH and FSH secretion.

phase decreases endothelial cell proliferation, blocks development of the extensive capillary bed, and interferes with normal luteal function, as judged by decreasing secretion of progesterone, presumably as a result of the reduction in the microvasculature. Similar results were reported in the PMS / hCG-treated rat (25). However, because in the rodent model the long-term anti-VEGF antibody treatment was started before initiation of follicular stimulation, it is difficult to separate interference with follicular maturation from luteal inhibition by the treatment. Yet, it is important to note that in our own data from the primate, short-term neutralization of VEGF during the follicular phase does not interfere with ovulation and corpus luteum function, as demonstrated by

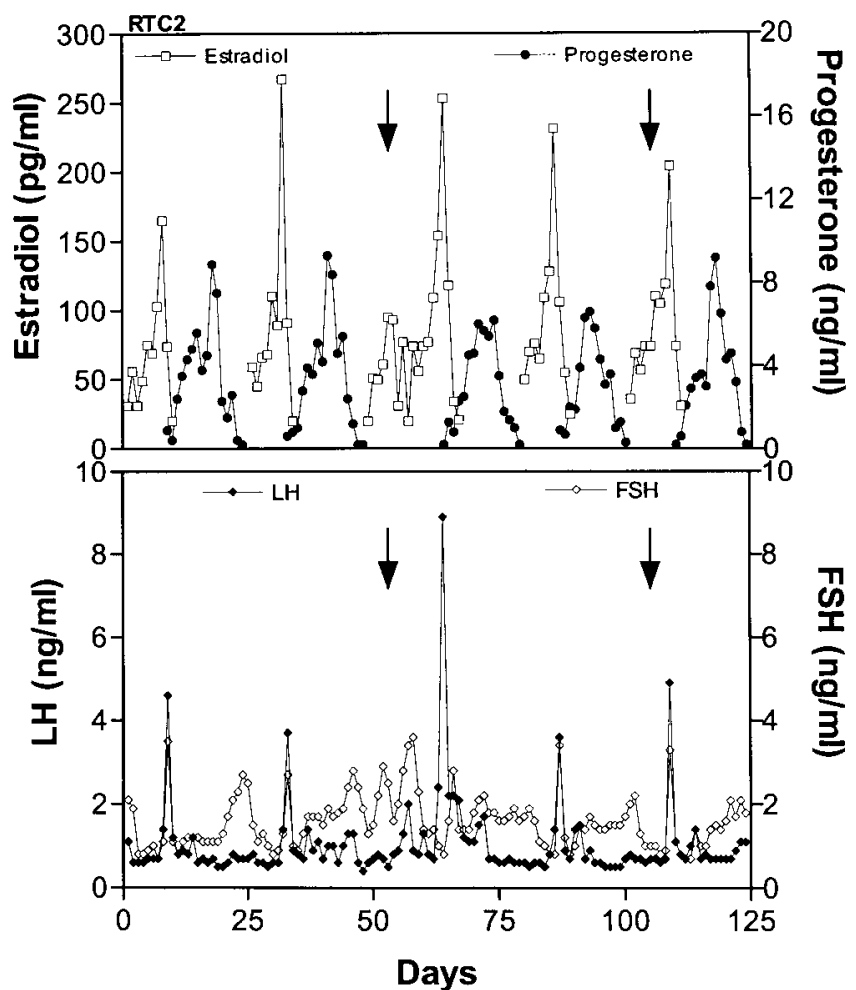

Fig. 3. Data from two successive control cycles, the anti-VEGF antibody-treated cycle, the posttreatment cycle, and the IgG-treated cycle in monkey RTC2 are presented. See Fig. 2 for details.

mean integrated luteal progesterone levels similar to those in the control menstrual cycles.

In conclusion, we have demonstrated that the angiogenic factor VEGF is a crucial physiological component in the process of follicular growth in the nonhuman primate. A significant delay in completion of the follicular phase occurs when VEGF activity is neutralized. Our data also indicate that the menstrual cycles that follow the treatment cycle are normal, suggesting that the inhibition of ovarian function by this antiangiogenic compound is temporary.

\section{Acknowledgments}

We thank the National Hormone and Pituitary Program (NIDDK) and Dr. A. F. Parlow (Harbor-University of California-Los Angeles Medical Center, Torrance, CA) for providing the reagents for monkey LH assays. We also acknowledge the help of Linna Xia-Zheng, Alinda Barth, and Nancy Cotui in performing the gonadotropin and steroid assays.

\section{References}

1. Folkman J, Shing Y. 1992 Angiogenesis. J Biol Chem. 267:10931-10934.

2. Reynolds LP, Redmer DA. 1992 Growth and microvascular development of the uterus during early pregnancy in ewes. Biol Reprod. 47:698-708.

3. Reynolds LP, Killilea SD, Redmer DA. 1992 Angiogenesis in the female reproductive system. FASEB J. 6:886-892.

4. Carmeliet P. 2000 Mechanisms of angiogenesis and arteriogenesis. Nat Med. 6:389-395.

5. Bates DO, Curry FE. 1996 Vascular endothelial growth factor increases hydraulic conductivity of isolated perfused microvessels. Am J Physiol. 271:H2520-H2528.

6. Ferrara N, Houck K, Jakeman L Leung DW. 1992 Molecular and biological properties of the vascular endothelial growth factor family of proteins. Endocr Rev. 13:18-32.

7. Ferrara N, Davis-Smyth T. 1997 The biology of vascular endothelial growth factor. Endocr Rev.18:4-25. 
8. Koos RD. 1993 Ovarian angiogenesis. In: Adashi EY, Leung PCK, eds. The ovary. New York: Raven Press; 433-453.

9. Zeleznik AJ, Schuler HM, Reichert Jr LE. 1981 Gonadotropin-binding sites in the rhesus monkey ovary: role of the vasculature in the selective distribution of human chorionic gonadotropin to the preovulatory follicle. Endocrinology. 109:356-362.

10. Ravindranath N, Little-Ihrig L, Phillips HS, Ferrara N, Zeleznik AJ. 1992 Vascular endothelial growth factor messenger ribonucleic acid expression in the primate ovary. Endocrinology. 131:254-260.

11. Otani N, Minami S, Yamoto M, et al. 1999 The vascular endothelial growth factor/fms-like tyrosine kinase system in human ovary during the menstrual cycle and early pregnancy. J Clin Endocrinol Metab. 84:3845-3851.

12. Yamamoto S, Konishi I, Tsuruta Y, et al. 1997 Expression of vascular endothelial growth factor (VEGF) during folliculogenesis and corpus luteum formation in the human ovary. Gynecol Endocrinol. 11:371-381.

13. Gordon JD, Mesiano S, Zaloudek CJ, Jaffe RB. 1996 Vascular endothelial growth factor localization in human ovary and fallopian tubes: possible role in reproductive function and ovarian cyst formation. J Clin Endocrinol Metab. 81:353-359.

14. Xiao E, Xia L, Shanen D, Khabele D, Ferin M. 1994 Stimulatory effects of interleukin-induced activation of the hypothalamo-pituitary-adrenal axis on gonadotropin secretion in ovariectomized monkeys replaced with estradiol. Endocrinology. 135:2093-2098.

15. Ferin M, Jewelewicz R, Warren M. 1993 The menstrual cycle: physiology, reproductive disorders and infertility. New York, Oxford: Oxford University Press.

16. Adashi EY. 1996 The ovarian follicular apparatus. In: Adashi EY, Rock JA, Rosenwaks Z, eds. Reproductive endocrinology, surgery, and technology. Philadelphia, New York: Lipincott-Raven; vol 1:17-40.
17. Goodman AL, Hodgen GD. 1983 The ovarian triad of the primate menstrual cycle. Recent Prog Horm Res. 39:1-73.

18. Christenson LK, Stouffer RL. 1997 Follicle-stimulating hormone and luteinizing hormone/chorionic gonadotropin stimulation of vascular endothelial growth factor production by macaque granulosa cells from pre- and periovulatory follicles. J Clin Endocrinol Metab. 82:2135-2142.

19. Hazzard TM, Molskness TA, Chaffin CL Stouffer RL. 1999 Vascular endothelial growth factor (VEGF) and angiopoietin regulation by gonadotrophin and steroids in macaque granulosa cells during the peri-ovulatory interval Mol Hum Reprod. 5:1115-1121.

20. Koos RD. 1995 Increased expression of vascular endothelial growth/permeability factor in the rat ovary following an ovulatory gonadotropin stimulus: potential roles in follicle rupture. Biol Reprod. 52:1426-1435.

21. Kettel LM, Roseff SJ, Chiu TC, et al. 1991 Follicular arrest during the midfollicular phase of the menstrual cycle: a gonadotropin-releasing hormone antagonist imposed follicular-follicular transition. J Clin Endocrinol Metab. 73:644-649.

22. Ditkoff EC, Cassidenti DL, Paulson RJ, et al. 1991 The gonadotropin-releasing hormone antagonist (Nal-Glu) acutely blocks the luteinizing hormone surge but allows for resumption of folliculogenesis in normal women. Am J Obstet Gynecol. 165:1811-1817.

23. Balmaceda JP, Schally AV, Coy D, Asch RH. 1981 The effects of an LH-RH antagonist ([N-Ac-D-Trp(1)(3), D-p-Cl-Phe(2), D-Phe(6), D-Ala(10)]-LH-RH) during the preovulatory period of the rhesus monkey. Contraception. 24:275-281.

24. Fraser HM, Dickson SE, Lunn SF, et al. 2000 Suppression of luteal angiogenesis in the primate after neutralization of vascular endothelial growth factor. Endocrinology. 141:995-1000.

25. Ferrara N, Chen H, Davis-Smyth T, et al. 1998 Vascular endothelial growth factor is essential for corpus luteum angiogenesis. Nat Med. 4:336-340. 